\title{
EchoGéo
}

$56 \mid 2021$

Les dynamiques spatiales contemporaines de la Russie

\section{Surprenante Russie}

Éditorial

\section{Serge Weber}

\section{(2) OpenEdition \\ Journals}

Édition électronique

URL : https://journals.openedition.org/echogeo/22008

DOI : $10.4000 /$ echogeo.22008

ISSN : 1963-1197

\section{Éditeur}

Pôle de recherche pour l'organisation et la diffusion de l'information géographique (CNRS UMR 8586)

\section{Référence électronique}

Serge Weber, «Surprenante Russie », EchoGéo [En ligne], 56 | 2021, mis en ligne le 25 juin 2021, consulté le 31 juillet 2021. URL : http://journals.openedition.org/echogeo/22008 ; DOI : https:// doi.org/10.4000/echogeo.22008

Ce document a été généré automatiquement le 31 juillet 2021.

EchoGéo est mis à disposition selon les termes de la licence Creative Commons Attribution - Pas d'Utilisation Commerciale - Pas de Modification 4.0 International (CC BY-NC-ND) 


\title{
Surprenante Russie
}

\author{
Éditorial
}

\section{Serge Weber}

1 La présente livraison d'EchoGéo forme un numéro dense, assorti de rubriques fournies, autour d'un dossier Sur le champ, coordonné par Sophie Hou et Yann Richard, offrant un panorama original des regards géographiques contemporains sur la Russie.

2 La rubrique Sur le vif propose deux textes qui viennent enrichir une année 2020-21 qui aura été féconde pour documenter le fil d'une actualité mouvementée. Christine Zumello propose une analyse cartographique fine et multiscalaire des résultats des élections de 2020 aux États-Unis. Les changements d'échelle d'observation et d'analyse qu'elle propose révèlent la complexité géographique des dynamiques du vote, tout en tenant compte des subtilités des découpages électoraux et des limites des interprétations cartographiques qu'ils induisent. Georgina André, Yu Qie et Etienne Monin nous livrent les résultats d'une enquête menée à Wuhan en plein confinement, dans la ville qui a été sur le front des mesures de restrictions sanitaires en Chine dès le début de la pandémie en janvier 2020. En choisissant le thème de l'approvisionnement alimentaire des habitants confinés, les auteurs dévoilent l'originalité des formes de structuration et d'encadrement de la vie locale à différentes échelles, les initiatives de solidarité de voisinage ainsi que la recomposition des rôles dans l'espace domestique.

3 Dans la rubrique Sur l'image, Olivier Bories, Pascal Fontorbes et Anne-Marie Granié restituent avec délicatesse une recherche filmique qu'ils ont menée auprès de paysans qui, en choisissant l'agroforesterie, contribuent à redessiner les paysages ruraux dans quelques communes du sud-ouest de la France. Leur approche sensible et intime, aux côtés des agriculteurs, parvient à mettre en lumière de beaux résultats, notamment sur le geste, le temps, le contact avec la parcelle.

4 La rubrique Sur l'écrit propose une interview faite par Nacima Baron de Gilles Rabin autour de son livre Berlin est une gare, publié cette année. L'entretien explore les différentes facettes du lien très fort qu'entretient le tissu urbain berlinois avec le chemin de fer et les gares. Les observations subtiles de l'auteur sont enrichies d'une 
épaisseur historique et de réflexions documentées sur les dimensions culturelle, sociale et phénoménologique de la gare emblématique de Friedrichsstrasse.

5 La rubrique Sur le métier est riche de deux textes, l'un qui est indépendant du dossier thématique, sur l'usage pédagogique de Wikipédia, l'autre qui se rattache au thème du dossier Sur le champ et traite de la géographie du VIH et du Sida en Russie. C'est en tant que pédagogue qu'Hadrien Commenges nous propose une réflexion à partir d'expérimentations pédagogiques sur la manière d'utiliser Wikipédia dans l'enseignement à l'université. Il détaille avec précision et inventivité différents outils et ressources permettant aux étudiants de mieux s'approprier Wikipédia et propose des pistes originales pour les accompagner vers un usage fécond et leur faire découvrir les enjeux du web sémantique. Avec le texte de Daniel Mathieu, nous entrons dans le cœur du numéro : la Russie d'aujourd'hui. Il nous fait part de son expérience d'expert, en tant que conseiller pour les affaires sociales à l'Ambassade de France en Russie et investi dans les coopérations entre la France et la Russie dans la prévention et la lutte contre le $\mathrm{VIH} /$ sida. Il nous apporte un regard éclairé sur les freins à la mise en œuvre de la prévention et sur les inégalités sociales et géographiques que met au jour cette situation sensible en Russie, ce qui fait de ce texte un texte où la part de témoignage fait écho aux pistes de recherches développées au sein de ce champ.

6 Le dossier thématique de la rubrique Sur le champ rassemble des textes qui témoignent de la vivacité de la recherche actuelle en géographie sur la Russie, attentive à la fois aux effets des transformations profondes qui ont reconfiguré les espaces à toutes les échelles depuis le début des années 1990 et aux contradictions qui apparaissent entre des orientations politiques affirmées qui touchent tant les régions centrales que les marges éloignées et des initiatives habitantes d'appropriation et de mobilisation qui sont habituellement peu documentées.

7 Deux premiers articles font d'emblée leur place aux métropoles secondaires, qui ont été beaucoup moins représentées que Moscou et Saint-Pétersbourg dans ce qu'on peut lire en France de la géographie urbaine russe. Ekaterina Mikhailova choisit un certain nombre d'indicateurs pour comparer le degré d'internationalisation des capitales régionales, en excluant les deux premières métropoles du pays. Ses analyses, portant sur des variables nombreuses, telles que la propriété foncière, les jumelages, les chaînes d'hôtels internationales, les FMN, les représentations diplomatiques, etc., aboutissent à une typologie confirmant des trajectoires métropolitaines nettement différenciées dans le processus d'internationalisation trente ans après la dissolution de l'URSS. Camille Robert-Boeuf choisit Kazan et la focale des datchas et des jardins hérités de la période soviétique pour aborder les effets de la métropolisation dans les villes secondaires. Ces collectifs de jardins, situés dans des espaces intersitiels rendus stratégiques par la croissance urbaine aux yeux des acheteurs et des promoteurs, sont révélateurs des inégalités socio-spatiales et de l'apparition de mécanismes de ségrégation. Le droit à la nature vient décliner de façon originale le droit à la ville dans les agglomérations postsoviétiques.

8 La géographie des mobilisations et des initiatives habitantes de réappropriation face à de grandes décisions d'aménagement se développe, comme ailleurs, en Russie. Guénola Inizan présente son étude de Moscou au prisme d'un programme de rénovation urbaine lancé par la municipalité et ciblant le parc des immeubles dits "khrouchtchéviens ", autant de quartiers de blocs qui, après avoir constitué un front d'extension urbaine et une ressource importante pour l'accès au logement des 
Moscovites, se retrouve, depuis la considérable expansion spatiale de l'agglomération, dans une situation à fort potentiel de rentabilisation. Les projets de démolitions et de relogements ont été accompagnés d'une concertation et ont fait l'objet de mobilisations habitantes qui révèlent à la fois les divergences des positionnements, selon les trajectoires résidentielles des habitants, et les profonds bouleversements de la stratification sociale moscovite depuis le changement de régime. Perrine Poupin explore le marché des déchets, opaque et peu régulé en Russie, à partir du cas d'un projet de décharge de déchets ménagers moscovites dans le grand Nord, dans la région d'Arkhangelsk, un milieu humide et fragile, isolé, un "espace déchet». Les mobilisations des habitants, inattendues dans un espace aussi peu densément peuplé, ne sont pas sans lien avec l'entretien de la mémoire du peuplement, remontant aux déportations. Elles s'organisent contre une forme de waste colonialism, dans une région déjà éprouvée par la pollution des activités extractives. Contre toute attente, la relégation territoriale et les grandes distances ont rendu possible l'émergence d'un type nouveau de mouvement collectif sur les réseaux sociaux.

On ne saurait dresser un portrait de la Russie aujourd'hui sans aborder le cyberespace et ses enjeux politiques. Hugo Estecahandy défriche un objet de recherche difficile d'accès. Il s'intéresse aux facteurs de localisation d'une activité qui échappe habituellement aux raisonnements géographiques, les fermes de minage de cryptomonnaies en Sibérie, dans la région d'Irkoutsk. Celles-ci bénéficient de la conjonction de plusieurs contextes favorables: l'offre abondante de foncier dans les friches industrielles, la bas coût de l'électricité lié à la surproduction électrique régionale, une localisation sur la dorsale de fibre optique Europe-Asie, la marginalisation de la région des principaux technoparcs informatiques sibériens, sans oublier l'héritage historique d'une culture libertaire. Kevin Limonier, en s'intéressant à la souveraineté numérique, pose la question de l'articulation entre cyberespace et territoire. Il propose une cartographie de l'Internet russe, reposant sur les traces numériques, les pouvoirs topologiques et l'articulation entre les données numériques et l'espace concret, pour comprendre comment la complexité des infrastructures spatialisées d'Internet mettent au défi les tentatives du pouvoir fédéral d'en accentuer le contrôle et d'y établir une frontière numérique.

10 Autant de textes qui invitent à s'immerger dans les espaces et les pratiques des habitants ordinaires qui ont dû s'habituer à beaucoup d'extraordinaire dans leur vie au cours des dernières décennies. 